Original

\title{
Efectos en el tiempo de la reubicación laboral y la calidad de vida en trabajadores mineros con silicosis de la División Andina-Codelco, Chile
}

\author{
The over time effects of work relocation on the quality of life in mine workers with \\ silicosis in the Andean division of Codelco, Chile
} \author{
Liliana Parra Osorio ${ }^{4}$, Óscar Ramírez Yerba ${ }^{5}$, Raquel González Baltazar ${ }^{2}$. \\ 1. Unidad Salud Ocupacional, Clínica Río Blanco. Los Andes. Chile. \\ 2. Universidad de Guadalajara. Guadalajara. México. \\ 3. Clínica del Trabajador. Manta. Ecuador. \\ 4. Universidad Libre de Cali. Cali. Colombia. \\ 5. Clínica del Trabajador. Lima. Perú. \\ Recibido: 28-09-11 \\ Aceptado: 03-10-11

\section{Correspondencia} \\ Diemen Delgado García, MD., MPH., Ph.D. \\ Unidad Salud Ocupacional. \\ Clínica Río Blanco S.A. \\ Av. Santa María 777, Villa Minera Andina. Los Andes (Chile). \\ Tfno.: (56)034-590547 \\ E-mail: ddelgado@crbcodelco.cl
}

Diemen Delgado García ', José Salazar Estrada², María de los Ángeles Aguilera 2, Fabián Delgado García ${ }^{3}$,

Resumen

Introducción: Los países industrializados están sufriendo importantes transformaciones en su evolución demográfica, caracterizado por el envejecimiento de la población (disminución de la tasa de natalidad, incremento de la población adulta y aumento de la esperanza de vida). En España el decremento de la tasa pensionista/ cotizante conlleva a que en la actualidad se esté discutiendo en el gobierno el retraso en la edad de jubilación.

Objetivo: Determinar los efectos en el tiempo de la reubicación laboral y la calidad de vida de los trabajadores mineros con diagnóstico de silicosis de la División Andina, Codelco Chile.

Materiales y Método: Estudio cuantitativo de series temporales en un solo grupo, se aplicó el cuestionario SF 36 a cinco trabajadores con diagnóstico de silicosis, tres meses antes de la reubicación y tres meses después de la reubicación, las mediciones fueron mensuales, para el análisis estadístico se utilizó el ANOVA para medidas repetidas en SPSS y se complementó con el test de Friedman.

Resultados: Se observó que en las tres mediciones antes de la reubicación laboral las 8 dimensiones del cuestionario SF 36 disminuyeron sostenidamente, no existiendo diferencia significativa (f $>0.05)$ entre los sujetos del estudio. Sin embargo después de la reubicación laboral la dimensión de dolor corporal, vitalidad y función social presentaron diferencia significativa $(\mathrm{f}<0.05)$ entre los sujetos del estudio.

Conclusiones: Los trabajadores mostraron cambios en el tiempo en la calidad de vida, respecto a la reubicación laboral, a pesar que no hubo significancia estadística en la mayoría de las dimensiones que se midieron durante el proceso de reubicación.

Med Segur Trab (Internet) 2011; 57 (225) 339-347

Palabras claves: Silicosis, Reubicación Laboral, Calidad de Vida, Trabajadores Mineros. 


\section{Abstract}

Objective: To determine the effects over time of work relocation and the quality of life of mine workers diagnosed withsilicosis in the Andean division of Codelco, Chile.

Materials and Method: Quantitative study of temporal series ina single group. The SF36 questionnaire was applied to five workers diagnosed with silicosis, three months before relocation and three months after job relocation. The evaluations were monthly. The statistical analysis was done using ANOVA for repeated measures in SPSS and complemented with the Friedman test.

Results: We observed that in all three measurements beforethe job relocation, the 8 dimensionsof the SF36 declined steadily, with no significant difference ( $>>0.05$ ) among the study subjects. However, after the redeployment, the dimension of body pain, vitality and social function showed significant differences $(\mathrm{f}<0.05)$ among the study subjects.

Conclusions: Workers showed changes over time in quality of life, with respect to job relocation, although there was no statistical significance in mostof the dimensions that were measured during the relocation process.

Med Segur Trab (Internet) 2011; 57 (225) 339-347

Key words: Silicosis, Job Relocation, Quality of Life, Miners. 


\section{INTRODUCCIÓN}

El concepto de reubicación laboral en Chile, bajo el amparo del Seguro Social de Accidentes del Trabajo y Enfermedades Profesionales ${ }^{1}$, Ley 16.744 , en su Art. $71,1^{\text {er }}$. Inciso, estipula "los trabajadores afectados de alguna enfermedad profesional deberán ser trasladados, por la empresa donde presten sus servicios, a otras faenas donde no estén expuestos al agente causante de la enfermedad".

La reubicación, desde el aspecto legal, favorece al trabajador, al no seguir expuesto, al agente que le ocasionó la enfermedad. Sin embargo, desconocemos qué ocurre en el trabajador minero, con silicosis, en cuanto a su calidad de vida, en el proceso mismo de la reubicación laboral.

Este estudio se desarrolló en trabajadores mineros con silicosis de la División Andina-Codelco Chile, ya que es la única empresa donde se cumple el proceso de reubicación laboral.

Es importante también mencionar que, la enfermedad pulmonar (silicosis), en la etapa de diagnóstico, motivo de la reubicación laboral, es completamente asintomática ${ }^{2}$, por lo que no guarda relación con características propias de otras enfermedades pulmonares.

La Silicosis es una enfermedad pulmonar fibro-génica bien conocida ${ }^{3}$. Se origina por la inhalación del dióxido de sílice en su forma de cristal.

La radiografía de tórax con técnica OIT, bajo los estándares de la Organización Internacional del Trabajo ${ }^{4}$, es el método diagnóstico utilizado para detectar silicosis ${ }^{5}$.Una vez realizada la radiografía, debe ser leída por un lector entrenado, comparándola con las placas patrón de la OIT 2000 de acuerdo a la siguiente nomenclatura: pequeñas opacidades regulares (p,q,r) y profusión en una escala de 12 niveles $(0 /-, 0 / 0,0 / 1,1 / 0,1 / 1,1 / 2,2 / 1$, $2 / 2,2 / 3,3 / 2,3 / 3$ y $3 /+)$.

La evaluación de la carga mundial de enfermedades y lesiones ocupacionales es difícil obtenerla ${ }^{6}$, la información fiable para la mayoría de los países en desarrollo, es escasa, principalmente, debido a graves limitaciones en el diagnóstico de las enfermedades ocupacionales y en los sistemas de reporte.

La Organización Mundial de la Salud, calcula que en América Latina, por ejemplo, sólo se reportan entre 1 y 5\% de todas las enfermedades ocupacionales ${ }^{7}$.

La Organización Internacional del Trabajo ${ }^{8}$, calcula que el $37 \%$ de los mineros de América Latina tienen silicosis, porcentaje que asciende al 50\% para los mayores de 50 años. En Chile las regiones más afectadas son la II, III, V, Metropolitana, VI y la VIII.

En Chile, no existe información suficiente que permita mostrar la real situación de salud de los trabajadores ${ }^{9}$, debido a un importante problema de sub-diagnóstico y subnotificación de enfermedades, accidentes y muertes por el trabajo ${ }^{10}$, lo cual obedece, principalmente, a la falta de capacitación en salud ocupacional de los equipos de salud y a la inexistencia o escasez de especialistas en medicina del trabajo ${ }^{11}$.

De ahí nuestro interés de realizar una investigación, para conocer los efectos en el tiempo que la reubicación laboral provoca en la calidad de vida del trabajador minero con silicosis de la División Andina-Codelco, Chile.

\section{MATERIALES Y MÉTODO}

Estudio cuantitativo de series temporales en un solo grupo, Se seleccionaron a los trabajadores mineros con silicosis, cuyo diagnóstico se realizó entre enero a abril del 2010. 


\section{Criterios de inclusión}

Trabajadores de la empresa minera División Andina, con diagnóstico profesional por silicosis, con reubicación confirmada durante el periodo de enero a abril de 2010 que aceptaron participar en el estudio.

\section{Criterios de exclusión}

1. Trabajadores contratados por otras empresas

2. Trabajadores con sospecha de silicosis que no estén diagnosticados como enfermedad profesional.

3. Trabajadores que ya hubieran sido reubicados previamente al inicio del trabajo de campo.

\section{Criterios de eliminación}

Trabajadores que no hayan contestado el cuestionario SF 36, en forma completa, en cualquiera de los momentos de evaluación.

\section{Procedimiento}

Se utilizó el siguiente criterio de evaluación: mínimo tres observaciones previas a la reubicación y tres posteriores a la reubicación. Las evaluaciones fueron mensuales.

\section{Descripción general de captación de información}

Para la captación de información se utilizó el cuestionario SF 36 Versión Española ${ }^{12}$.

\section{Prueba piloto}

El objetivo fue evaluar la aplicación del cuestionario SF 36 Versión Española 1.4, a un grupo de trabajadores reubicados por enfermedades osteomusculares de la División Andina. Este ejercicio nos sirvió para modificar o ratificar las preguntas de acuerdo al modismo chileno. En conclusión, el cuestionario SF 36, fue comprendido por los trabajadores reubicados por enfermedades ósteo-musculares.

\section{Análisis de datos}

Las variables que caracterizan a la población de estudio, como edad, antigüedad, se presentan con su media (DS).La categoría laboral se presentan con su frecuencia (absoluta y relativa).

Para la prueba de hipótesis se empleó el estadístico ANOVA ${ }^{13}$, para medidas repetidas en SPSS (StatisticalPackagefor Social Sciences) v 13.0. En el estudio, se tomó más de una medición de una misma característica, en cada sujeto, por lo que comparamos la media de la misma variable medida en tres ocasiones, antes y después, de la reubicación a los mismos sujetos.

Además se aplicó el test de Friedman ${ }^{14}$,ya que el tamaño de la muestra fue pequeño.

Se informó a cada participante seleccionado y voluntario del estudio, el objetivo específico del mismo. No se realizó ninguna intervención o modificación intencionada en los individuos. Sólo se trabajó con cuestionarios. Por lo tanto, éste fue un estudio de riesgo mínimo.

No obstante, el autor, se comprometió a manejar la información bajo las normas establecidas por el Código Internacional de Ética Médica ${ }^{15}$. 


\section{RESULTADOS}

En este estudio dirigido a mostrar los efectos en el tiempo de la reubicación laboral, en la calidad de vida de trabajadores con silicosis, participaron cinco sujetos.

Todos los trabajadores incluidos, tenían más de 20 años de antigüedad en la empresa, que es el tiempo de exposición identificado en la población mundial para iniciar con silicosis crónica. La edad de los trabajadores que, con más frecuencia, se repite fue entre los 50 a 59 años de edad. Sin embargo, dos de ellos se encontraron en el rango de 40 a 49 años.

El estado civil de los cinco trabajadores, con diagnóstico de silicosis, en proceso de reubicación o reubicado, es casado. Respecto a la escolaridad, existió un abanico en cuanto a la adquisición de conocimientos formales en el sistema educacional chileno. No obstante, se observó un predominio en el segundo medio. Llamó la atención, un trabajador con silicosis, en proceso de reubicación o reubicado, con formación universitaria, ver tabla I.

Tabla I. Escolaridad de los trabajadores con silicosis, reubicados

\begin{tabular}{lcc}
\hline \multicolumn{1}{c}{ Nivel educacional } & Frecuencia & Porcentaje \\
\hline Primero medio & 1 & 20 \\
Segundo medio & 2 & 40 \\
Técnica & 1 & 20 \\
Universitaria & 1 & 20 \\
Total & 5 & 100 \\
\hline
\end{tabular}

Fuente: Directa.

En cuanto al puesto de trabajo y lugar de trabajo antes de la reubicación, todos ellos trabajaban expuestos a sílice, ocupación donde posiblemente adquirieron la enfermedad profesional, ver tabla II.

Tabla II. Puesto de trabajo, antes y después de la reubicación laboral

\begin{tabular}{lclc}
\hline \multicolumn{1}{c}{ Puesto de trabajo } & Frecuencia & Puesto de trabajo & Frecuencia \\
\hline Mantenedor mecánico & 1 & Gestor de proyectos & 1 \\
Operador de carguío & 2 & Analista de gestión & 3 \\
Operador de producción & 1 & Coordinador & 1 \\
Ingeniero en minería & 1 & Total & 5 \\
Total & 5 & & \\
\hline
\end{tabular}

Fuente: Directa.

En cuanto al puesto de trabajo después de la reubicación, todos ellos fueron alejados de la fuente que le generó la silicosis, ver tabla III.

Tabla II. Lugar de trabajo, antes y después de la reubicación laboral

\begin{tabular}{lclc}
\hline \multicolumn{1}{c}{ Lugar de trabajo } & Frecuencia & Lugar de trabajo & Frecuencia \\
\hline ConcentradorSAG & 1 & Proyectos & 1 \\
Mina subterránea N.16 & 2 & Bodega & 2 \\
Mina subterránea N. 17 & 2 & Servicios & 2 \\
Total & 5 & Total & 5 \\
\hline
\end{tabular}

Fuente: Directa. 
En las tres observaciones del estudio de los cinco sujetos, antes de la reubicación laboral, se observó que la media muestral de las ocho dimensiones del cuestionario SF 36, disminuyó sostenidamente, en los trabajadores mineros con silicosis, en proceso de reubicación, no existiendo diferencia significativa como mostró la f (Friedman) intergrupos, en las ocho dimensiones del cuestionario SF 36, cuyo valor fue > a 0.05 como se muestra en la tabla IV.

Tabla IV. Media muestral respecto al efecto en el tiempo, antes de la reubicación laboral

\begin{tabular}{lcrrrrrrrr}
\hline \multicolumn{2}{c}{ Dimensiones } & $\begin{array}{c}\text { Función } \\
\text { Física }\end{array}$ & $\begin{array}{c}\text { Rol } \\
\text { Físico }\end{array}$ & $\begin{array}{c}\text { Dolor } \\
\text { Corporal }\end{array}$ & $\begin{array}{c}\text { Salud } \\
\text { General }\end{array}$ & Vitalidad & $\begin{array}{c}\text { Función } \\
\text { Social }\end{array}$ & $\begin{array}{c}\text { Rol } \\
\text { Emocional }\end{array}$ & $\begin{array}{c}\text { Salud } \\
\text { Mental }\end{array}$ \\
\hline 1 Obs. & Media & 80 & 70 & 67 & 50 & 71 & 74.8 & 86.6 & 72 \\
2 Obs. & Media & 80 & 70 & 68,8 & 41 & 63 & 67.2 & 60 & 56 \\
3 Obs. & Media & 76 & 55 & 57.2 & 42.8 & 51 & 62.2 & 46.8 & 48.8 \\
& F & $\mathbf{0 . 0 8 3}$ & $\mathbf{0 . 1 7}$ & $\mathbf{0 . 2 4 5}$ & $\mathbf{2 . 2 8}$ & $\mathbf{0 . 6 9 7}$ & $\mathbf{0 . 2 8 8}$ & $\mathbf{1 . 0 4 4}$ & $\mathbf{2 . 0 1 2}$ \\
\hline
\end{tabular}

Fuente: Directa.

En las tres observaciones del estudio de los cinco sujetos, después de la reubicación laboral, se observó un aumento del puntaje en las dimensiones de función física, vitalidad, función social, salud mental y rol emocional, mientras que, el rol físico, dolor corporal y salud general aumentaron su puntaje al segundo mes de la reubicación pero disminuyó al tercer mes del fenómeno, no existiendo diferencia significativa como mostró la f (Friedman) inter-grupos, en cinco dimensiones del cuestionario SF 36, cuyo valor fue $>$ a 0.05. Sin embargo, en la dimensión dolor corporal, vitalidad y función social, sí existe significancia mostrando una $\mathrm{f}<\mathrm{a} 0.05$ como se muestra en la tabla V.

Tabla V. Media muestral respecto al efecto en el tiempo, después de la reubicación laboral

\begin{tabular}{lrrrrrrrrr}
\hline \multicolumn{2}{c}{ Dimensiones } & $\begin{array}{c}\text { Función } \\
\text { Física }\end{array}$ & $\begin{array}{c}\text { Rol } \\
\text { Físico }\end{array}$ & $\begin{array}{c}\text { Dolor } \\
\text { Corporal }\end{array}$ & $\begin{array}{c}\text { Salud } \\
\text { General }\end{array}$ & Vitalidad & $\begin{array}{c}\text { Función } \\
\text { Social }\end{array}$ & $\begin{array}{c}\text { Rol } \\
\text { Emocional }\end{array}$ & $\begin{array}{c}\text { Salud } \\
\text { Mental }\end{array}$ \\
\hline 4 Obs. & Media & 79 & 70 & 63.2 & 43.4 & 55 & 77.6 & 40 & 52.8 \\
5 Obs. & Media & 83 & 90 & 67 & 47.8 & 58 & 77,6 & 40 & 57.6 \\
6 Obs. & Media & 83 & 70 & 61.2 & 45 & 58 & 80.2 & 60 & 64.8 \\
& F & $\mathbf{0 . 0 8 9}$ & $\mathbf{0 . 4 4 4}$ & $\mathbf{0 . 0 4}$ & $\mathbf{0 . 1 0 6}$ & $\mathbf{0 . 0 2 2}$ & $\mathbf{0 . 0 1 1}$ & $\mathbf{0 . 2 2 2}$ & $\mathbf{0 . 4 8 4}$ \\
\hline
\end{tabular}

Fuente: Directa.

Al realizar el análisis de las seis observaciones del estudio de los cinco sujetos, se observó que en la dimensión función física y función social, existe un efecto beneficioso durante el proceso mismo de la reubicación laboral.

Lo contrario ocurrió con el dolor corporal, salud general, vitalidad, rol emocional, salud mental que se vieron afectados por el proceso mismo de la reubicación laboral.

Mientras que la dimensión que no reflejó modificación, en el puntaje, fue el rol físico, no existiendo diferencia significativa como mostró la f (Friedman) inter-grupos, en 
las ocho dimensiones del cuestionario SF 36, cuyo valor fue > a 0,05 como se muestra en la tabla VI.

Tabla VI. Media muestral respecto al efecto en el tiempo, proceso de reubicación laboral

\begin{tabular}{lrrrrrrrrr}
\hline \multicolumn{2}{c}{ Dimensiones } & $\begin{array}{c}\text { Función } \\
\text { Física }\end{array}$ & $\begin{array}{c}\text { Rol } \\
\text { Físico }\end{array}$ & $\begin{array}{c}\text { Dolor } \\
\text { Corporal }\end{array}$ & $\begin{array}{c}\text { Salud } \\
\text { General }\end{array}$ & Vitalidad & $\begin{array}{c}\text { Función } \\
\text { Social }\end{array}$ & $\begin{array}{c}\text { Rol } \\
\text { Emocional }\end{array}$ & $\begin{array}{c}\text { Salud } \\
\text { Mental }\end{array}$ \\
\hline 1 Obs. & Media & 80 & 70 & 67 & 50 & 71 & 75.2 & 86.66 & 72 \\
2 Obs. & Media & 80 & 70 & 68,8 & 41 & 63 & 67.8 & 60 & 56 \\
3 Obs. & Media & 76 & 55 & 57.2 & 42.8 & 51 & 62.8 & 46.72 & 48.8 \\
4 Obs. & Media & 79 & 70 & 63.2 & 43.4 & 55 & 77.6 & 40 & 52.8 \\
5 Obs. & Media & 83 & 90 & 67 & 47.8 & 58 & 77.6 & 40 & 57.6 \\
6 Obs. & Media & 83 & 70 & 61.2 & 45 & 58 & 80.2 & 60 & 64.8 \\
\hline & F & $\mathbf{0 . 1 1 2}$ & $\mathbf{0 . 3 3 5}$ & $\mathbf{0 . 1 0 2}$ & $\mathbf{0 . 3 9 6}$ & $\mathbf{0 . 3 4 5}$ & $\mathbf{0 . 2 6 8}$ & $\mathbf{0 . 6 3 2}$ & $\mathbf{0 . 9 7 7}$ \\
\hline
\end{tabular}

Fuente: Directa.

\section{DISCUSIÓN}

Esta investigación, nos muestra los efectos en el tiempo que presentaron los trabajadores con silicosis de la División Andina, en cuanto a la reubicación laboral y la calidad de vida, como algunas coincidencias, respecto a la aparición de la silicosis después de la exposición de varios años a sílice, en la radiografía de tórax con técnica OIT, que es entre los 20 a 25 de exposición a sílice ${ }^{16}$.

Al realizar inferencia con los datos obtenidos en nuestra investigación, la $\mathrm{f}$ de Friedman fue $>$ de 0,05 por lo que no existió asociación, probablemente, esto ocurrió por los siguientes factores:

1. La muestra es pequeña, tan sólo cinco trabajadores con silicosis, en proceso de reubicación.

2. El tiempo de estudio fue muy reducido, de enero a abril del 2010.

3. Al ser sólo cinco trabajadores, probablemente, cada individuo, influyó su respuesta del cuestionario $\mathrm{SF} 36$, de acuerdo a su personalidad.

Se observó que las dimensiones que componen el cuestionario SF 36, y su respuesta en el tiempo de estos trabajadores, nos pueden entregar conocimiento del fenómeno estudiado, al evidenciar fluctuaciones antes y durante el proceso de reubicación.

En nuestro estudio, los trabajadores, narran que sus competencias, no son abordadas para la toma de decisión de la reubicación y del nuevo puesto de trabajo. En Colombia se estudia las competencias residuales de trabajadores, donde se toman en cuenta, las competencias del trabajador para proceder a la reubicación ${ }^{17}$.

A pesar de que en Chile, la silicosis, produce incapacidad por normativa de la Ley 16.744, ésta no se manifiesta clínicamente en las primeras etapas. Sin embargo, el proceso mismo de la reubicación, altera los ámbitos personales y sociales, esto debido principalmente a la espera que tienen los trabajadores y a la angustia de no saber en qué lugar serán reubicados. La incapacidades y/o /invalidez en las enfermedades respiratorias,son de indudable repercusión personal y social ${ }^{18}$.

La dimensión de salud general, es la más baja engatillada por la incertidumbre de la silicosis y motivo de la reubicación por la enfermedad. Los resultados en estas dimensiones, son similares al estudio de la calidad de vida en pacientes con diagnóstico de enfermedad pulmonar obstructiva crónica, evidenciando una valoración baja, en la dimensión de salud general ${ }^{19}$. 
En nuestra investigación, no se estudió la depresión como tal, en los trabajadores reubicados con silicosis pero, las dimensiones más afectadas, fueron la salud general y el rol emocional. En un estudio sobre calidad de vida en mujeresdeprimidas en el postparto, se observó que dimensiones más afectadas, fueron el rol emocional, el rol físico y la vitalidad ${ }^{20}$.

En pacientes con diagnóstico de hiperplasia prostática, se estudia la calidad de vida, encontrándose que la dimensión de función física, fue la más alta, mientras que la dimensión de salud general obtuvo una valoración pobre o negativa ${ }^{21}$. En nuestro estudio, los resultados en estas dimensiones son similares.

En un estudio que relaciona la función pulmonar y calidad de vida en pacientes con miastenia gravis ${ }^{22}$, la dimensión más afectada fue la actividad física.En nuestro estudio, la función pulmonar, no está afectada por la silicosis, por lo que la función física, no presentó modificación, ni antes, ni después, de la reubicación laboral.

En pacientes con diagnóstico de tuberculosis pulmonar, se estudia la calidad de vida, en la dimensión dolor corporal, se aprecia que los pacientes tuvieron poca interferencia en el dolor en sus actividades de trabajo y de hogar ${ }^{23}$. En nuestro estudio el de dolor corporal fue importante, sin existir relación orgánica de síntomas causales ya que, la silicosis, sólo produce sintomatología, en etapas avanzadas de la enfermedad.

\section{CONCLUSIONES}

A pesar de ser un grupo pequeño de trabajadores mineros con silicosis reubicados, respecto a las características socio-demográficas y laborales, se logró identificar que, tanto jefes, como empleados, se ven afectados por esta enfermedad, la cual no está influenciada por edad, ni escolaridad, pero sí, por las áreas de trabajo, con exposición a sílice.

Los resultados del estudio, están de acuerdo también al constructo, al encontrar diferencias individuales, que se pueden explicar por el soporte social de los compañeros de trabajo y familia. Ambos, pudiesen generar fenómenos individuales, los cuales van a depender de las características, capacidades, objetivos y oportunidades del individuo, en el proceso de adaptación.

\section{RECOMENDACIONES}

En base a las conclusiones, se sugiere elaborar políticas locales, empresariales y públicas encaminadas a adoptar acciones preventivas en la industria minera que eviten la silicosis.

Se sugiere mantener esta investigación en el tiempo y conocer qué ocurre con esta población a mediano plazo y, por supuesto, agregar nuevos casos de trabajadores para formar una cohorte y que este diseño nos dé más luces del fenómeno estudiado.

\section{AGRADECIMIENTOS}

A Irma Patricia Cano Páez, amiga, esposa y compañera, por su dedicación al cuidado de Diemen Dampier y Ashley Militza durante estos tres años de distracción y alejamiento de mi núcleo familiar, al sumergirme en esta investigación, facilitándome el tiempo y el espacio de concentración, cuyo único objetivo será aportar al conocimiento humano. 


\section{REFERENCIAS BIBLIOGRÁFICAS}

Ministerio del Trabajo y Previsión Social, (1968, 01 de Febrero). Seguro Social Contra Riesgos de Accidentes del Trabajo y Enfermedades Profesionales, Ley 16.744. Extraído el 27 de diciembre del 2008 desde http://www.leychile.cl/N?i=28650\&f=2008-12-27\&p=

1. Abú K, Fanlo P, Lorente MP. Silicosis. Anales del Sistema Sanitario de Navarra 2005; 28: 83-9.

2. Hornung V, Bauernfeind F, Halle A, Samstad E, Kono H, Rock K, Fitzgerald K, Latz E. Silica crystals and aluminum salts activate the NALP3 inflammasome through phagosomal destabilization. NatureInmunology 2008; 9: 847-56.

3. International Labour Organization. International Classification of Radiographs of Pneumoconiosis (The complete Set of Radiographs). Geneva: ILO; 2000.

4. Fraser R, Paré J. Diagnosis of diseases of the chest. Philadelphia: WB Saunders Company; 1990.

5. Organización Mundial de la Salud. Salud de los trabajadores: proyecto de plan de acción mundial (Informe de la Secretaria A60/20). Ginebra: 60ª Asamblea Mundial de la Salud; 2007.

6. World Health Organization. Global strategy on occupational health for all. The way to health at work (Document WHO/OCH/95.1). Geneva:WHO; 1994.

7. Organización Internacional del Trabajo. Información sobre Seguridad en el Trabajo. Ginebra: Juan Somavia, Director General de la OIT; 2005.

8. Bernales B, Alcaino J, Solís R. Situación de exposición laboral a sílice en Chile. Ciencia \& Trabajo 2008 ; 10: 1-6.

9. Pan American Health Organization. Halth in the Americas (Scientific Publication No. 569). Washington, DC: PAHO Library Cataloguing in Publication Data; 1998

10. Vallebouna C. Las Enfermedades profesionales: Un olvido de la salud pública. El Vigía 2003; 6: 26-30

11. Alonso J, Prieto L, Antó JM. La versión española del SF-36 HealthSurvey (Cuestionario de Salud SF-36): un instrumento para la medida de los resultados clínicos. MedicinaClínica 1995; 104: 771-6.

12. Lumley T. Statistics in medicine:network meta-analysis for indirect treatment comparisons. John Wiley\&Sons, Ltd 2002; 21: 2313-24

13. Altman DG. Practical statistics for medical. London: Chapman and Hall; 1991.

14. Klimovsky E, Saidon P, Nudelman L, Bignone I. Declaration of Helsinki. Its vicissitudes durig the last five yeard. FundaciónRevistaMedicina 2002; 62: 365-70.

15. Seaton A, Legge JS, Henderson J,Kerr KM. Accelerated silicosis in scottish stonemasons. The Lance 1991; 337: 341-4.

16. Acosta C, Restrepo M. Enfoque integral de reubicación y readaptación laboral como respuesta al impacto de la violencia en los trabajadores colombianos. Revista Ciencias de laSalud 2004; 2: 62-4.

17. Montemayor T, Ortega F, Cejudo P, Sánchez H. Valoración de la capacidad laboral e incapacidad/ invalidez en las enfermedades respiratorias. ArchBronconeumol 2004; 40: 21-6.

18. Vinaccia S, Quiceno J, Zapata C, Obesso S, Quintero DC. Calidad de vida relacionada con la salud y emociones negativas en pacientes con diagnóstico de enfermedad pulmonar obstructiva crónica (EPOC). Psicología desde el Caribe Universidad del Norte 2006; 18: 89-108.

19. Rojas G, Fritsch R, Solís J, González M, Guajardo V, Araya R. Quality of life of women depressed in the post-partum period. Revista Médica de Chile 2006; 134: 713-20.

20. Vinaccia S, Quiceno J, Fonseca P, Fernández H. Calidad de vida relacionada con la salud y su impacto sobre la cognición hacia la enfermedad en pacientes con hiperplasia prostática. Acta Colombiana de Psicología 2006; 9: 47-56.

21. Freitas GA, Regiane V, Pradas J, Vigil L, Casan P. Relación entre función pulmonar y calidad de vida relacionada con la salud en la miastenia gravis generalizada. Archivos de Bronconeumología 2006; 42: 218-24.

22. Vinaccia S, Quiceno J, Fernández H, Pérez B, Sánchez M, Londoño A. Calidad de vida relacionada con la salud y apoyo social percibido en pacientes con diagnóstico de tuberculosis pulmonar. Anales de Psicología 2007; 23: 245-52

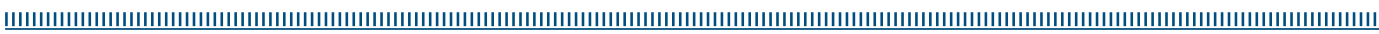

